\title{
An Optimization of Novel Hybrid Bilateral Filter for Isotropic and Anisotropic of 2D-Images in All kinds of Transmissions
}

\author{
J. Kishore Kumar ${ }^{1}$, Prof. S. Ramakrishna ${ }^{2}$, Dr. N. Naveen Kumar ${ }^{3}$ \\ ${ }^{1}$ Research Scholar, Department of Computer Science, Sri Venkateswara University, Tirupathi, India \\ ${ }^{2}$ Professor, Department of Computer Science, Sri Venkateswara University, Tirupathi, India \\ ${ }^{3}$ Assistant Professor, Department of Computer Applications, MITS, Andhra Pradesh, India
}

\begin{abstract}
In this paper, a new deblurring and denoising algorithm is proposed for isotropy and anisotropy reconstruction for 2Dimages using Novel Hybrid Bilateral Filter (NHBF) in low level to high level noise values. To overcome the drawback of traditional denoise filter mechanism, we used NHBF which will reconstruction an image from low abstracted to high abstracted pixel values. Experiment analysis reveals that our algorithm is efficient than other traditional algorithms like Mean Filter (MF) and Bilateral Filter (BF). This proposed method can be utilized in Satellite Image Processing, Atmospheric research Laboratories and Scientific Image Processing.
\end{abstract}

Keywords: Reconstruction, Bilateral Filter, Satellite Image Processing, Atmospheric research Laboratories and Scientific Image Processing.

\section{Introduction}

Generally images are of two types, one is Gray Scale Images and other one is RGB images. Gray scale image has only one channel and RGB image have three channels. Noise may occur due to heat of camera sensors, analogue to digital conversion and communication channels. This type of noises cause to degrade the image quality, therefore it is very important to remove the positive and negative impulses by using filtering technique. Most probably the Linear Filtering technique will consider the two filtering techniques like Mean Filter (MF) and Least Mean Square (LMS). Median Filter considers under non-liner filtering technique. Eliminating the noise process may contaminate the nature of an image in order to either improve its original visualization. Pixels corrupt at two intensity levels that is high or low intensity values. To identify the neighboring pixels Standard Median Filter (SMF) proposed in [1]. The SM filter was the first effective impulse noise removal filtering technique.

Speckle reducing anisotropic diffusion (SRAD) approach is derived based on partial differential equation (PDE) in [I iee imp] which will allows the generation of an image based on shape of the image and size of the window. The specific class of splitting-based finite, various theoretical and adequate methods, and additive operator splitting (AOS) approach are discussed in [1 ]. A two stage fuzzy filter approach for reduce heavy noise is presented in [2] , firstly; computes a fuzzy derivative for eight direction. In second; fuzzy derivatives to smoothing by weighting the neighboring pixels using pixel values. Impulse noise detectors and removal method is propose for Grayscal and Color Images are presented in [3]. A improved decision-base algorithm (DBA) is proposed in [4] which efficiently removes the salt and pepper noise in gray scale and color image preserving in detail. The DBA utilized previously processed neighboring pixel values to get better denoised in gray and color images. A new impulse noise removal approach for color image is presented in [5] work base on noise detection algorithm and weighted vector median filter. Additive white Gaussian noise (AWGN) process that is independent of the actual RGB values. Such approaches cannot effectively remove color noise produced by today's CCD digital camera. For this a new approach two phase unified framework for color noise which will work based on single piecewise smooth and noise level function (NLF) model is presented in [6]. Wavelet transforms are very powerful to remove noise suspicions in various wavelet based images. A wavelet based denoise method is presented in [7]. Edge detection is a process that detects the presence and location of edges constituted by sharp changes in color, intensity of an image. The image brightness is depending up on the depth of discontinuities of an image, surface orientation, different material properties and variations in scene. So it is a difficult task to remove the noise without eliminating the sharp characteristics of the image, such as edges, corners and other sharp structures during the denoising process. Edge detection is susceptible to noise. This is due to the fact that the edge detection algorithms are designed to respond to sharp changes, which are caused by noisy pixels. A new fuzzy filtering techniques for color image works for de-fuzzificaiton is presented in [8] which preserves any type of edges in any direction. Due to Haze in images, resulting contrast reduction and fading of color. To remove haze in images a new two stage approach is proposed in [9]. In first, denoise the image prior to dehazing this treats haze and noise separately, and second approach is denoise using dehaze of an iterative approach using adaptive and non-parametric method.

Speckle noise in images is causing difficulties for image understanding. Reduction of speckle noise is one of the most essential tasks in image preprocessing. An innovative 


\section{International Journal of Science and Research (IJSR) \\ ISSN (Online): 2319-7064}

Index Copernicus Value (2013): 6.14 | Impact Factor (2014): 5.611

approach to reduce speckle noise is presented in [10]. Image segmentation will play a major role to process segregating an image into different essential parts of object or regions. These regions share common characteristics based on color, intensity, texture, etc. segmentation includes edge detection , region growing methods, Clustering methods, Compression based methods, Histogram-based methods, etc. In image processing edge detection techniques are used to locate the borders of homogeneous region in an image depending on characteristics such as texture and intensity of image. To recover the original edges of an image an efficient approach called reconstructed color image segmentation using edge detection and threshold method is presented in [11]. A new method called YCoCg color Image Edge detection presented in [12]. Gray scale and color images impulse noise removal algorithm is presented in [13]. In the process of remove noise the image does not deteriorate. Reconstruction process is called the reconstruction of an image using decomposition or analysis of applied noised image. A new Image Decomposition Using Wavelet Transform is proposed in [14].

\section{Proposed Algorithm}

In this section we proposed Another filter computation, which will identify noise and after that displace noise corrupted pixel. Results demonstrate that the proposed channel produces fabulous results at various commotion levels.

Step 1: Read the input image and convert it into GRAY scale image.

Step 2: Select 2-D window of size $5 \times 5$ and assume the window size as $\mathrm{W}=3$

Step 3: Calculating the histogram bins (P) and cumulative sum of histogram $\operatorname{bins}(\Omega)$

Step 4: Calculate mean $(\mu x)$ by using the following equaiton

Mean $(\mu)=$ cumulative sum of histogram $\operatorname{bins}(\Omega)$ * number of bins in histogram of image.

Step 5: Calculate the sigma*mena by using the following equation

$$
\begin{gathered}
\sigma \text { or } \sigma \mathrm{x} \mu \text { "mu"or } \mu \mathrm{x}=\frac{(\sigma \mu * \Omega-\mu) 2}{\Omega * 1-\Omega} \\
\sigma \mathrm{x} * \mu \mathrm{x}=\frac{\left(\mathrm{c}(\mu+\Omega-\mu)_{2}\right.}{\Omega * 1-\Omega}
\end{gathered}
$$

where index=mean (maximum value of $(\sigma \mathrm{x} * \mu \mathrm{x})$ )

$\sigma=($ index-L)/(number of bins in histogram of image-L)

$\mathrm{L}$ is minimum to maximum pixel limits of input image

Step 6: Convert the image from RGB imge to LAB Image

Step 7: Apply the novel bilateral filter $F=H^{*} G$ (mininmum :: maximum limits)

Where $\mathrm{H}=$ exponential(-((dl) $2+(\mathrm{da}) 2+(\mathrm{db}) 2) /(2 *(\operatorname{sigma}) 2)$;

Here $\mathrm{l}$ is representing Lightness $\& \mathrm{a}$ and $\mathrm{b}$ are the color dimensions

$\mathrm{G}=$ Gaussian domain weight age values

Step 8: Apply the soft threshold for given image and convert it from LAB image to RGB image to get Novel Bilateral Filtered image.

Step 9: apply noval canny edge deteciton based on renyi entropy for 2D images to get better edge detection

$\mathrm{Q}=(1 /(1-\mathrm{alpha}))^{*} \log 2(\operatorname{sum}(\mathrm{P}, 2)+\mathrm{eps}) \quad$ E.q ... (3) Where $\mathrm{p}=$ The value for thresh is relative to the highest value of the gradient magnitude of the image

Alpha $=$ Shape Parameter
Eps = Floating Point Accuracy

Step 10: Round off these $Q$ values we are getting renyi entropy values

Step 11: renyi entropy maximum values are taken as threshold for canny edge detection and stop the procedure

\section{Simulation Results \& Discussion}

The simulation results are compared with traditional filters and proposed NHBF and shown in the Table 1, NHBF technique generating better results in high densities also. In table 2, generally we have shown the result based on PSNR values with general filter techniques. Figure1 shown the input image, figure 2 shows the MF simulation result, figure 3 shows the WF simulation result, figure 4 shows the proposed NHBF simulation result and figure 5 shows the performance of different filtering technique plot.

Table 1: The Mean, Wiener and NHBF comparison in terms

\begin{tabular}{|c|c|c|c|c|}
\hline Noise Level \% & Filter & $M S E$ & RMSE & PSNR \\
\hline \multirow[t]{3}{*}{10} & Mean & 1.3906 & 0.75 & 22.6503 \\
\hline & Wiener & 0.938 & 0.3062 & 34.3627 \\
\hline & NHBF & 0.0351 & 0.185 & 62.6768 \\
\hline \multirow[t]{3}{*}{20} & Mean & 2.668 & 1.0698 & 19.8206 \\
\hline & Wiener & 0.938 & 0.3062 & 34.3627 \\
\hline & NHBF & \begin{tabular}{|l|}
0.1066 \\
\end{tabular} & 0.2603 & 57.8515 \\
\hline \multirow[t]{3}{*}{30} & Mean & 8.375 & 1.6417 & 14.8526 \\
\hline & Wiener & 0.938 & 0.3062 & 34.3627 \\
\hline & NHBF & 0.2645 & 0.3173 & 53.9066 \\
\hline \multirow[t]{3}{*}{40} & Mean & 6.3711 & 1.6238 & 16.0403 \\
\hline & Wiener & 0.938 & 0.3062 & 34.3627 \\
\hline & $\mathrm{NHBF}$ & 0.5943 & 0.3699 & 50.3908 \\
\hline \multirow[t]{3}{*}{50} & Mean & 19.4102 & 2.3494 & 11.2021 \\
\hline & Wiener & 0.938 & 0.3062 & 34.3627 \\
\hline & NHBF & 0.9391 & 0.4107 & 48.4037 \\
\hline \multirow[t]{3}{*}{60} & Mean & 14.1875 & 2.183 & 12.5633 \\
\hline & Wiener & 0.938 & 0.3062 & 34.3627 \\
\hline & $\mathrm{NHBF}$ & 1.3109 & 0.4454 & 46.9552 \\
\hline \multirow[t]{3}{*}{70} & Mean & 31.6992 & 2.6693 & 9.0719 \\
\hline & Wiener & 0.938 & 0.3062 & 34.3627 \\
\hline & NHBF & 1.9035 & 0.4836 & 45.3354 \\
\hline \multirow[t]{3}{*}{80} & Mean & 34.0234 & 2.5981 & 8.7646 \\
\hline & Wiener & 0.938 & 0.3062 & 34.3627 \\
\hline & NHBF & 2.56 & 0.5155 & 44.0483 \\
\hline \multirow[t]{3}{*}{90} & Mean & 41.2031 & 2.7049 & 7.9331 \\
\hline & Wiener & 0.938 & 0.3062 & 34.3627 \\
\hline & NHBF & 3.2803 & 0.5466 & 42.9717 \\
\hline
\end{tabular}
of MSE, RMSE and PSNR

Table 2: Result \& Comparison of mean, Wiener and proposed NHBF Filtering technique

\begin{tabular}{|c|c|c|c|c|}
\hline $\begin{array}{c}\text { Noise } \\
\text { Level \% }\end{array}$ & $\begin{array}{c}\text { Mean Filter } \\
\text { - PSNR }\end{array}$ & $\begin{array}{c}\text { Wiener Filter - } \\
\text { PSNR }\end{array}$ & $\begin{array}{c}\text { Proposed - } \\
\text { NHBF PSNR }\end{array}$ & $\begin{array}{c}\text { Noise } \\
\text { Level \% }\end{array}$ \\
\hline 10 & 22.6503 & 34.3627 & 62.6768 & 10 \\
\hline 20 & 19.8206 & 34.3627 & 57.8515 & 20 \\
\hline 30 & 14.8526 & 34.3627 & 53.9066 & 30 \\
\hline 40 & 16.0403 & 34.3627 & 50.3908 & 40 \\
\hline 50 & 11.2021 & 34.3627 & 48.4037 & 50 \\
\hline 60 & 12.5633 & 34.3627 & 46.9552 & 60 \\
\hline 70 & 9.0719 & 34.3627 & 45.3354 & 70 \\
\hline 80 & 8.7646 & 34.3627 & 44.0483 & 80 \\
\hline 90 & 7.9331 & 34.3627 & 42.9717 & 90 \\
\hline
\end{tabular}




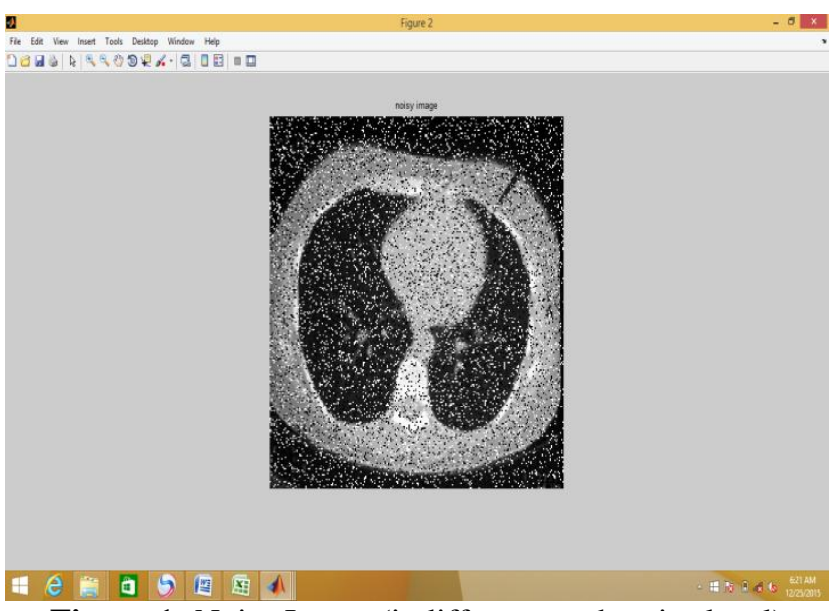

Figure 1: Noise Image (it differs at each noise level)

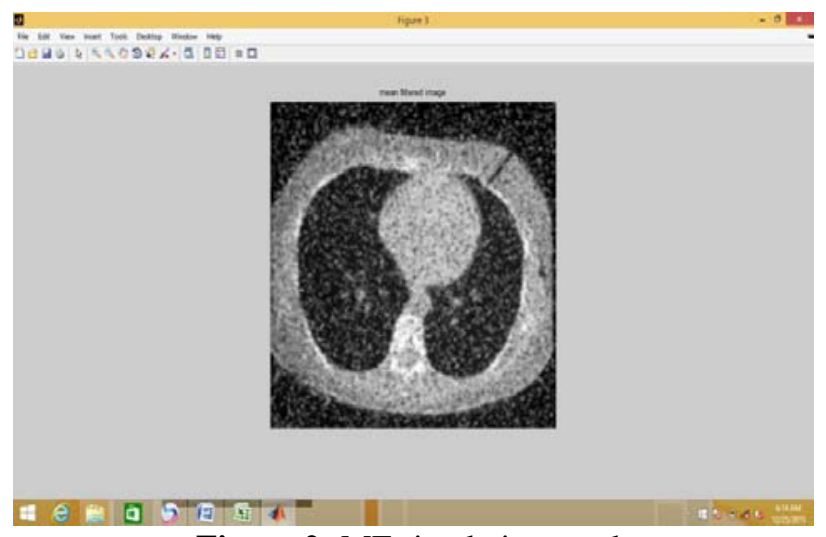

Figure 2: MF simulation result

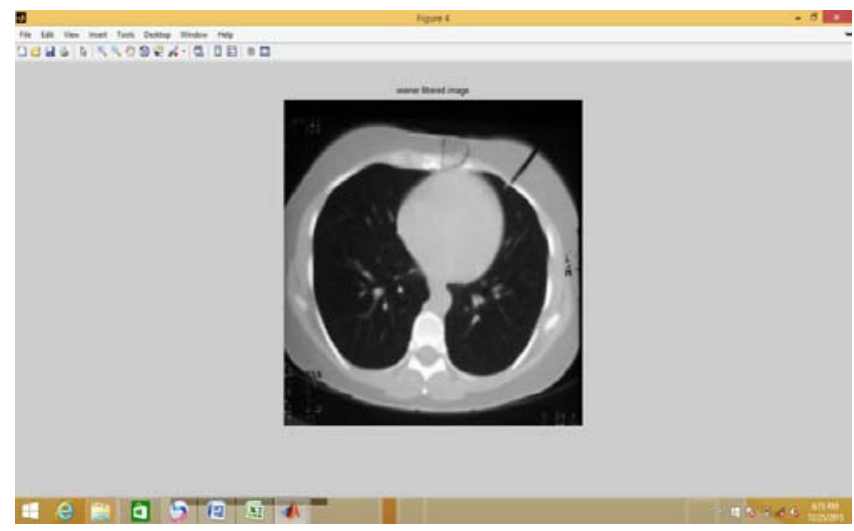

Figure 3: WF simulation result

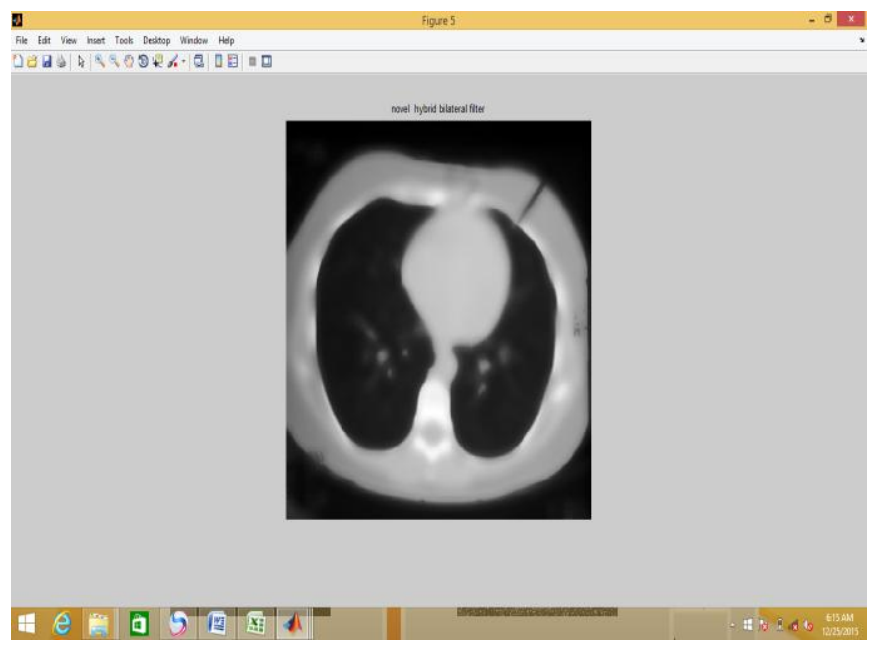

Figure 4: proposed NHBF simulation result

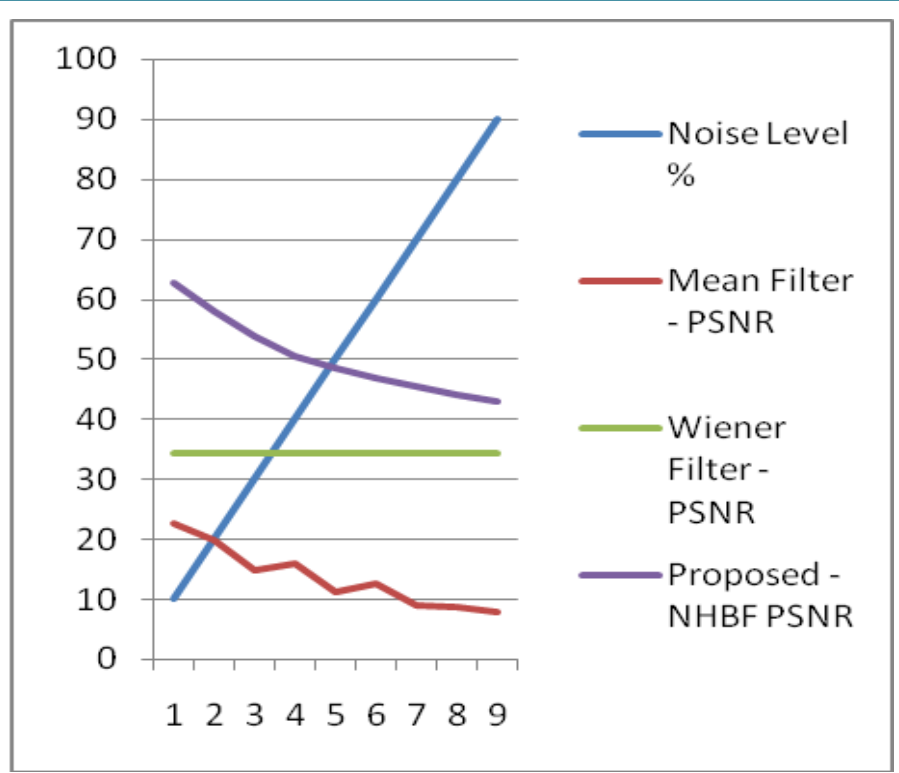

Figure 5: plot for differentiation of MF, WF and NHBF

\section{Conclusion}

The proposed NHBF, fabricate that an successful lossless images in all noise values for isotropic and anisotropic of 2D-Images. This filtering mechanism will work in different hierarchy of pixels in all dimensions and the results are compared with the traditional filtering techniques like MF and BF with the characteristics of PSNR, MSE and RMSE it proves that NHBF work more robotic than traditional filters at all noise values.

\section{References}

[1] J. Astola and P. Kuosmanen, Fundamentals of Nonlinear Digital Filtering. Boca Raton, FL: CRC, 1997R. Caves, Multinational Enterprise and Economic Analysis, Cambridge University Press, Cambridge, 1982. (book style)

[2] Tao Chen, Kai-Kuang Ma, and Li-Hui Chen, "Tri-State Median Filter for Image Denoising”, IEEE Transactions on image processing, 1999, pp 1834-1838

[3] Pitas and A. N. Venetsanopoulos, Nonlinear Digital Filters: Principles and Applications. Boston, MA: Kluwer, 1990

[4] T. Sun and Y. Neuvo, "Detail-preserving median based filters in image processing," Pattern Recognit. Lett., vol. 15, pp. 341-347, Apr. 1994.

[5] C.Ni, Q.Li and L.Z.Xia, "A Novel Method of Infraed Image Denoising and Edge Enhancement," Proc.of Cong. on Image and Signal Procssing, vol.3,2008

[6] Y.Lin,X.Zhou, L.Song, "Applicaton of Contourlet Transform in Infrared Image Denoising," Proc. Infrared Materials, Devices, and Applications, vol.6835,2008.

[7] M.Cheng, X.Mei, J.Lin, L.Wang, "Infrared Image Denoising Method based on Improved C-HMT Model," 7th Int.Conf.on System Simulation and Scientific Computing, 2008.

[8] C.Ni, L.Xia, "Nonsubsampled Pyramid based Adaptive Anisotropic Diffusion Filtering for Infrared Image Denoising," 6th Int. Conf. on Wireless Communications Networking and Mobile Computing, 2010. 
[9] Z.Xiaok, J.Shi, Z.Guan, "Infrared Image Denoising Based on Statanary Wavelet Transform," Int. Conf. on Digital Image Processing. In Proc.SPIE 7546, 2010.

[10] B.Zhan, Y.Wu, "Infrared Image Enhancement Based on Wavelet Transform and Retinex," Int. Conf. On Intelligent Human- machine System and Cybernetics, 2010.

[11]K.Choi, C.Kim, and J.B. Ra,"Infrared Image Enhancement Based onan Aligned High Resolution Visible Image," Proc. of 17th ICIP,2010.

[12] Hossein Rabbani, "Image denoisingin steerable pyramid domain basedon a local Laplace prior," pattern Recognitaion, bol.42, no.9, pp. 2181-2193,2009.

[13] Dr. N. Naveen Kumar, Chendra Sekhar et.al Simplified filtering technique to edge reconstruction and comparison of original image in low to high density noise values, International Journal of Science and Research (IJSR), ISSN (Online): 2319-7064 Volume 3 Issue 8, August 2014.

[14]Dr. N. Naveen Kumar, J.Kishore Kumar Reddy et.al Gray Scale Image Edge Detection and Reconstruction Using Stationary Wavelet Transform in High Density Noise Values, International journal of computer engineering \& Applications, ISSN 2321-3469, Vol.II, Issue I/III, 2013 